\title{
The calculation basis for a four-component jet mixer for fertilizer and water
}

\author{
Sergey Andreyevich Tarasyants, ${ }^{1,}$, Vladimir Alexandrovich Rudakov ${ }^{1}$, Yulia Sergeyevna \\ Urzhumova $^{1}$, and Karina Alexandrovna Degtiareva ${ }^{2}$ \\ ${ }^{1}$ Novocherkassk Engineering and Meliorative Institute named after A.K. Kortunov - Don State Uni- \\ versity Branch, Novocherkassk, Russian Federation \\ ${ }^{2}$ South Russian State Polytechnic University (NPI) named after M.I. Platonov, Novocherkassk, Rus- \\ sian Federation
}

\begin{abstract}
This article covers calculation basis for a four-component jet mixer for fertilizer and water. The calculation for a four-component jet mixer for fertilizer consists in determining the basic geometric and hydraulic parameters of a four-component jet mixer for fertilizer and water and supplying the mixture to irrigated areas, with fertilizing irrigation, which will allow supplying all types of fertilizers and changing the concentration of mixture in the required proportions. All geometric relative parameters are calculated on the basis of the need to scale the mixer dimensions and are taken as the ratio of the diameters of the mixer elements to the cylindrical part of the mixing chamber. The above calculation bases determine the main parameter of the mixer necessary for determining all the elements the cross-sectional area of the annular nozzle. It was founded that when calculating the geometric characteristic, you should be guided by the desire to accept the value of the geometric characteristic $m=2.5 \div 8.0$, corresponding to the maximum value of the efficiency up to $40 \%$ and higher.
\end{abstract}

\section{Introduction}

The most common methods of mixing fertilizers and water, during fertilizing irrigation, are mixing tanks, injectors for applying fertilizers, devices mounted on a hydrant of an irrigated area, tanks for preparing mother solution, trailed spreaders, etc.

The analysis of devices in mixing systems shows that the above devices are complicated in design and inconvenient in operation. This publication covers calculation basis for a four-component jet mixer for fertilizer and water (figure 1,2) [1].

Calculation of a four-component jet mixer for fertilizer consists of determining the optimal relative geometric and hydraulic parameters of the mixer for the possibility of designing and manufacturing its elements when applying fertilizers to a given area in case of fertirrigation.

\footnotetext{
*Corresponding author: starasyancz@mail.ru
} 


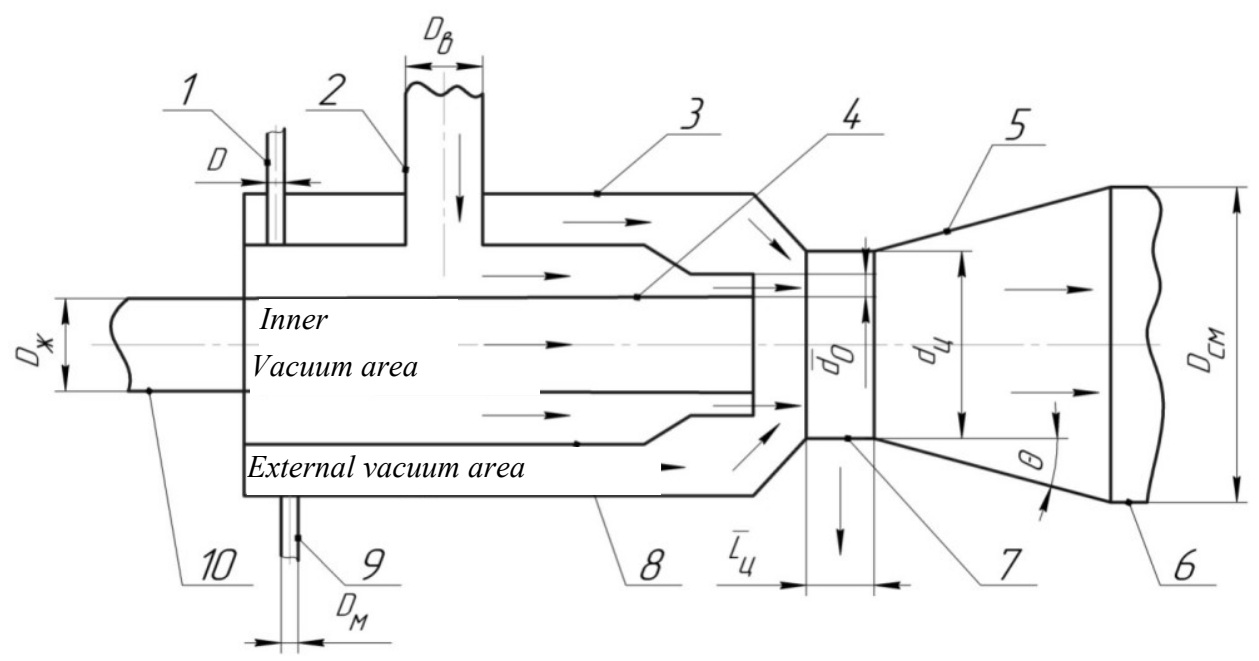

Fig. 1. Structural diagram of a four-component jet mixer for fertilizer.

Were: 1 - mineral fertilizers supply pipeline; 2 - working water supply pipeline; 3 - outside case of the mixer; 4 - inner adapter of the annular nozzle; 5 - diffuser; 6 - pipeline for discharging a mixture of fertilizers and water; 7 - mixing chamber; 8 - outer adapter of the annular nozzle; 9 - microelements supply pipeline; 10 - pipeline for supplying livestock waste.

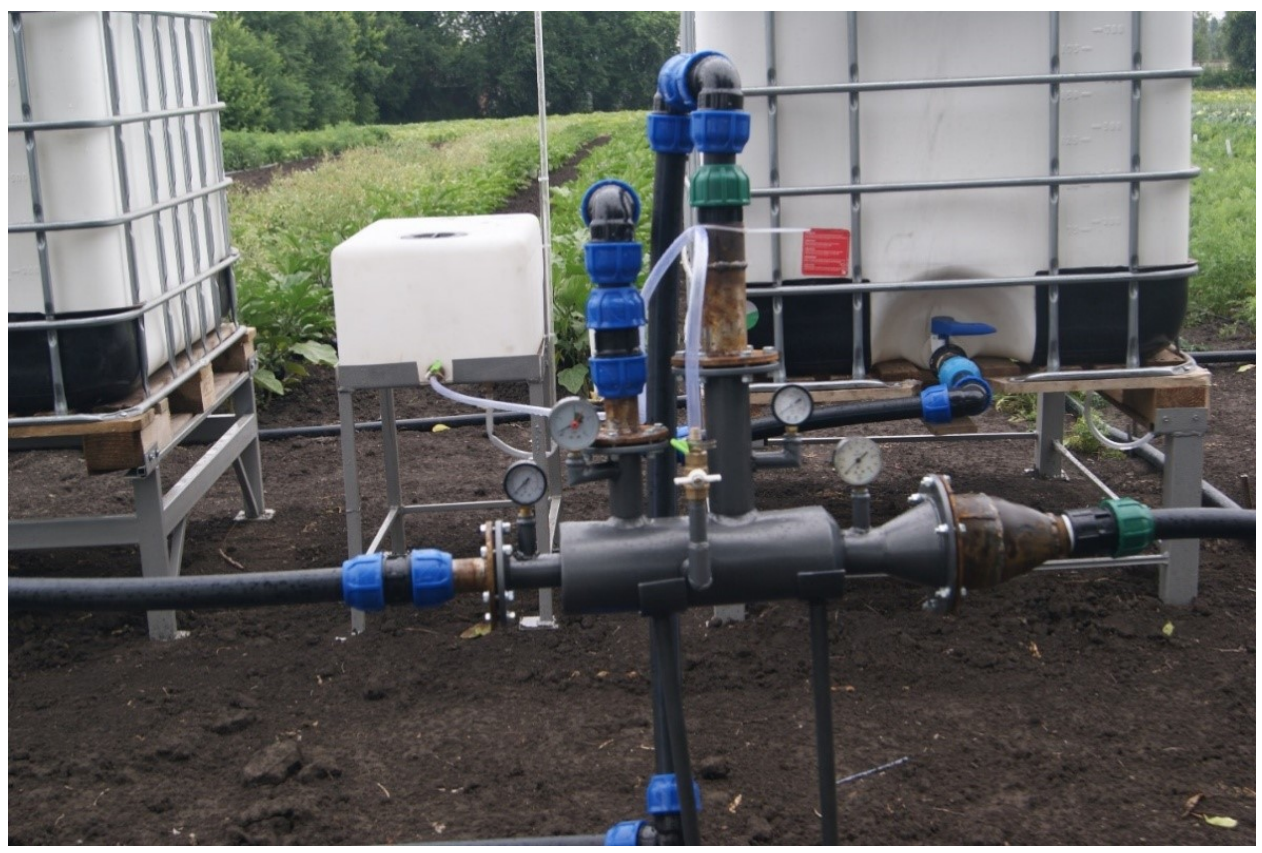

Fig. 2. Four-component jet mixer Overall view. 


\section{Materials and Methods}

The geometric parameters of the mixer include:

- the relative outer $\bar{d}_{0}^{\prime}$ and inner diameters $\bar{d}_{0}^{\prime \prime}$ of the annular nozzle adapters;

- the relative distance from the nozzle edge to the beginning of the cylindrical part of the mixing chamber $\bar{Z}$;

- the actual diameter of the mixing chamber $d_{t s}$;

- the relative length of the mixing chamber $\bar{L}_{u}$;

- the actual opening angle of the diffuser $\theta$;

- diameters of supplying water pipelines $D_{w}$, mineral fertilizers $D_{m}$, livestock sewage $\mathrm{D}_{\mathrm{l}}$, a mixture of fertilizers and water $\mathrm{D}_{\mathrm{fw}}$;

$-m$ is the geometric characteristic of the mixer (the ratio of the cross-sectional area of the mixing chamber to the cross-sectional area of the annular nozzle end).

All geometric relative parameters are calculated on the basis of the need to scale the mixer dimensions and are taken as the ratio of the diameters of the calculated mixer elements to the cylindrical part of the mixing chamber.

Hydraulic parameters include:

- pump head of the water blower $\mathrm{H}_{\mathrm{w} . b l}$;

- flow rate in the nozzle $V_{0}$;

- kinetic energy in the nozzle $V_{0}^{2} / 2 g$;

- Flow rate in the mixing chamber $V_{c}$;

- kinetic energy in the mixing chamber $K_{c}=\frac{V_{c}^{2}}{2 g}$;

- total energy in the mixing chamber $\frac{p_{c}}{g \rho_{c}}$;

- the amount of vacuum (pressure) in the mixing chamber $\frac{p_{b}}{g \rho_{c}}\left(\frac{p_{c}}{g \rho_{c}}\right)$;

- water flow consumption $Q_{0}$ in the nozzle;

- sucked fertilizer consumption in the nozzle $Q_{1}$;

- mixing ratio $\alpha_{0}=\frac{Q_{1}}{Q_{0}}$.

\section{Results and Discussion}

The main parameter affecting the mixer operation is the cross-sectional area of the annular nozzle $\omega_{0}=0,785\left[d_{0}^{\prime^{2}}-d_{0}^{/ / 2}\right]$, which has the main effect on the outgoing flow rate of the working jet (water) (Figure 3). 


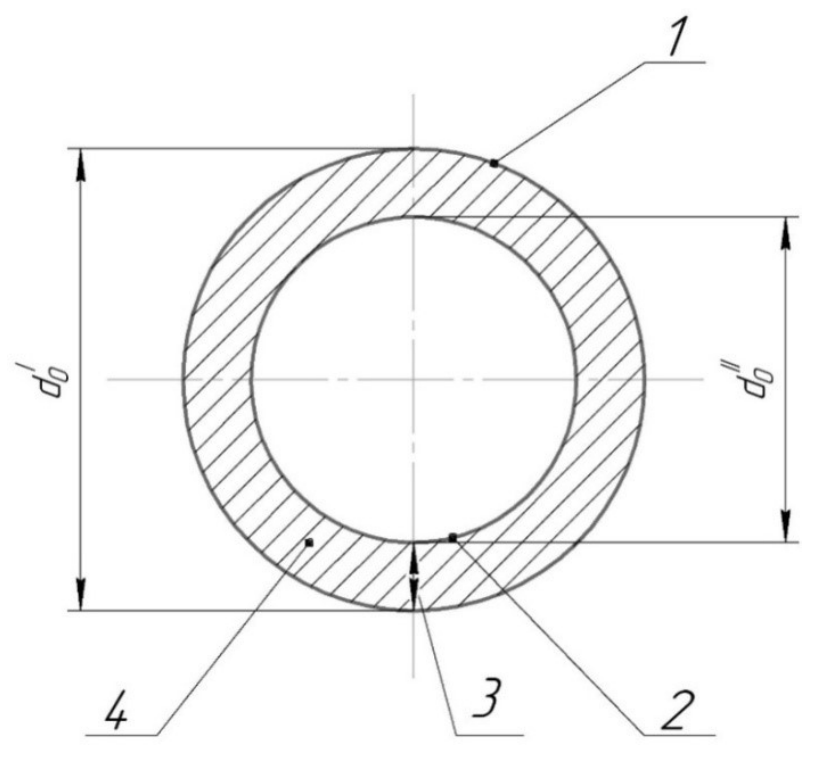

Fig. 3. Cross-sectional diagram of the nozzle end.

Were: 1 - inner diameter of the outer nozzle; 2 - outer diameter of the inner nozzle; 3 - the width of the slot $\mathrm{h}$ in the nozzle; 4 - outlet area of the working stream (water)

The main parameter, the kinetic energy in the mixing chamber, is determined according to the annular nozzle outlet flow rate $V_{0}$. The main principle for calculating the diameters $d_{0}^{\prime}$ and $d_{0}^{\prime \prime}$ is the assignment of the slot width in the nozzle and the possibility of the presence of such a working flow rate that can create the vacuum necessary for fertilizers suction. The diameter of the outer adapter $d_{0}^{\prime}$ is assigned in the amount of 0.9-0.95 the diameter of the mixing chamber $d_{t s}$ [2]. The outer diameter of the inner adapter $d_{0}^{/ /}$(Figure 4) influences a lot - the width of the slot h, the flow rate in the nozzle $V_{0}$, the working flow supply $Q_{0}$, the flow rate in the mixing chamber $V_{c}$, the mixing ratio $\alpha_{0}$. The inner adapter outer diameter of the annular nozzle $d_{0}^{/ /}$plays a key role in the operation of the entire jet mixer. The required calculation is given below.

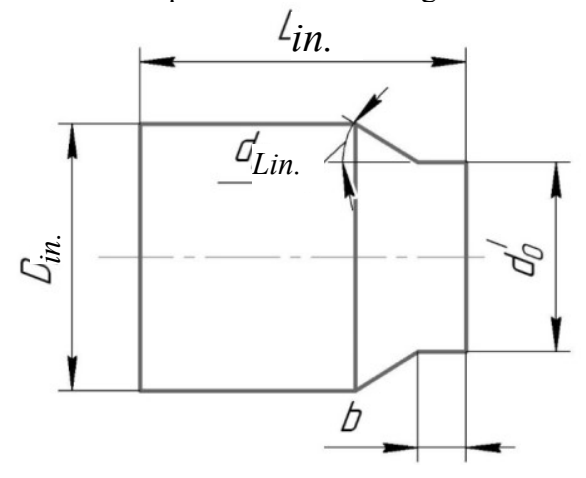

a)

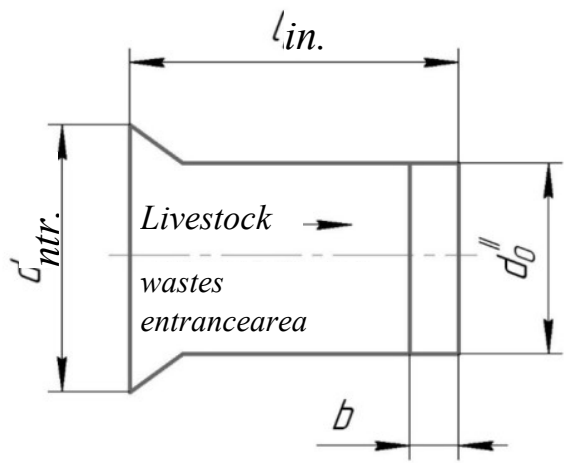

b)

Fig. 4. Diagram of the external and internal adapters of the annular nozzle. 
Were: a - external annular nozzle; b - internal annular nozzle

- the width of the slot $h$, if possible, is taken as the maximum and is determined by hydraulic calculations;

- the end length of the cylindrical sharpening is taken in the amount of $0.1-0.15$ diameter and plays a passive role in the mixer operation;

- the length $l_{\text {int }}$ is determined taking into account the possibility of inserting the fertilizer supply pipelines into the mixer case; it is taken as short as possible to reduce the pressure loss at the inlet $\varsigma_{\text {in }}$ of livestock sewage and mineral fertilizers;

- The inlet diameter $d_{\text {in }}$ to the inner surface of the adapter is taken as the maximum possible based on the diameter of the external case of the mixer.

The relative distance from the edge of the annular nozzle to the beginning of the cylindrical part of the mixing chamber $\bar{Z}$ (Figure 5) is taken from the literature data as $0.1 \div$ $0.2 d_{\mathrm{ts}}[3]$.

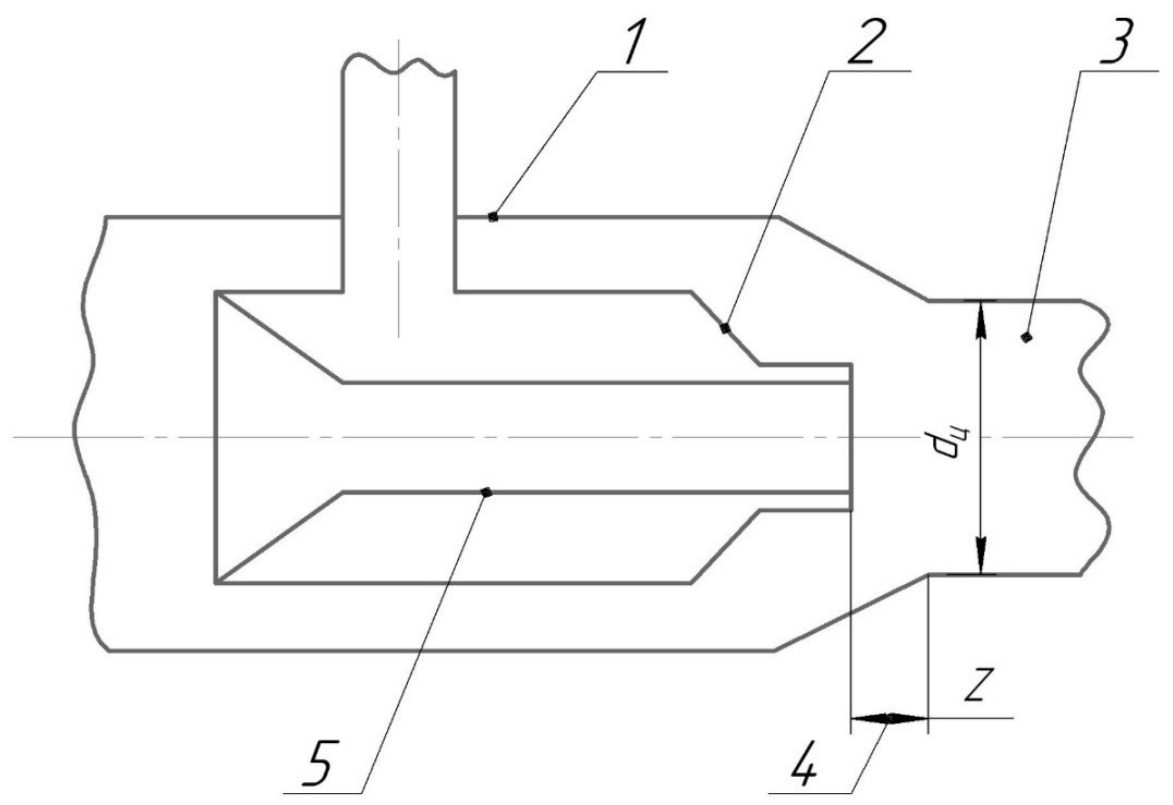

Fig. 5. Scheme of binding the mixing chamber to the edge of the annular nozzle.

Were: 1 - case; 2 - outer adapter of the annular nozzle; 3 - mixing chamber; 4 - distance from the nozzle edge to the beginning of the cylindrical part of the mixing chamber; 5 - inner adapter of the annular nozzle

In fact, the diameter of the mixing chamber $d_{t s}$ also depends on many factors, and basically all other diameters of the mixing chamber in their final form are bound to it. The flows are mixed in the mixing chamber, the velocity diagram of the working and suction jet is leveled, according to the energy losses in the mixing chamber, the efficiency of the entire mixer is determined and the amount of vacuum required for fertilizers suction is determined. The calculation of the mixing chamber diameter is mainly based on the condition of the presence of such a value of kinetic energy in it, which is capable of creating the maximum value of negative pressure. The length of the mixing chamber determines the main energy losses in the mixer due to the presence of the maximum speed of the two flows. The design of the proposed annular two-surface four-component mixer [4] offers the shortest 
possible length of the mixing chamber due to the presence of a concave velocity curve diagram [5] (Figure 6).

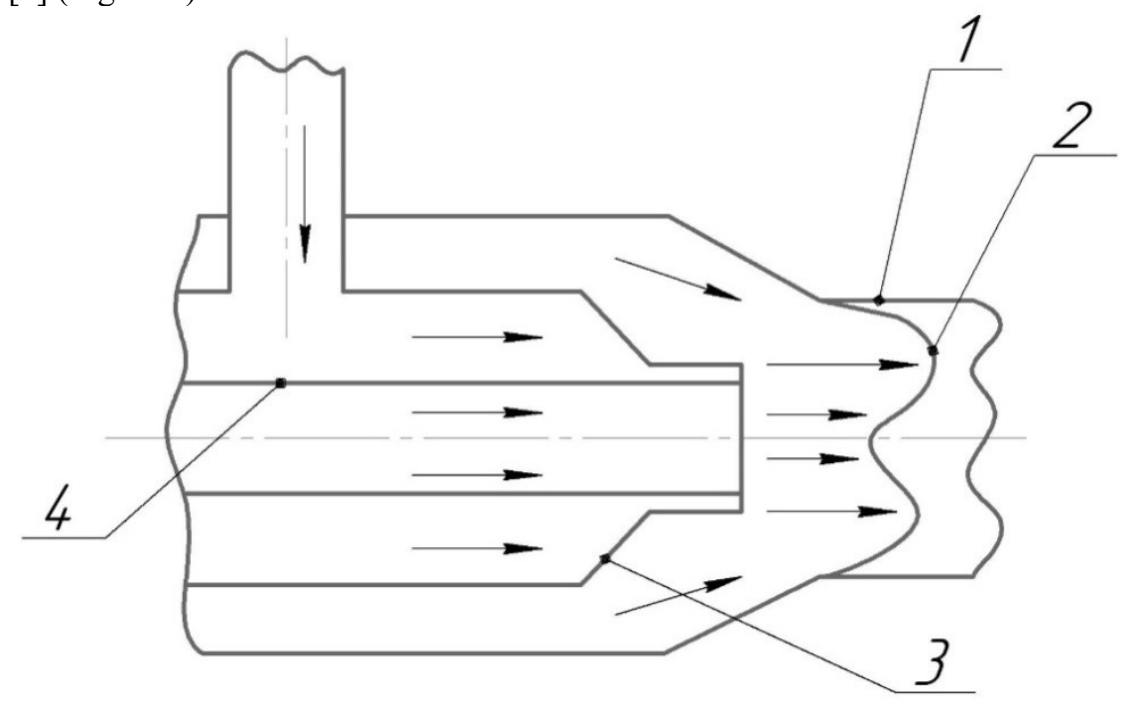

Fig. 6. Schematic diagram of the velocity curve diagram in the mixing chamber of the mixer.

Were: 1 - mixing chamber; 2 - concave velocity curve diagram in the mixing chamber; 3 - outer adapter; 4 - inner adapter

When determining the expansion angle value of the diffuser, you should proceed from two conditions:

- the requirement of reducing the diffuser length (reduce the length of the entire installation);

- reducing the coefficient of hydraulic resistance, reducing losses and, accordingly, increasing the efficiency of the mixer.

The above mentioned two conditions depend on one factor - the expansion angle, which, based on the literature data [6], corresponds to $6-10^{\circ}$ for the smallest pressure losses.

When determining the diameters of pipelines, you should be guided by the known value of the relative flow rate in pressure pipelines, corresponding to $2-2.5 \mathrm{~m} / \mathrm{s}$ [6].

When calculating the geometric characteristics $\omega_{0}$ (the ratio of the cross$m=\frac{\omega_{u}}{\omega_{0}}$ (the ratio of the cross-
ional area of the annular nozzle), you sectional area of the mixing chamber to the cross-sectional area of the annular nozzle), you
should be guided by the desire to accept such a value that contributes to an increase in the efficiency of the mixer. Optimal values of the geometric characteristic $m$ for jet mixers are taken in the range of $2.5 \div 8$ and depends on the jet unit pattern of use. For fertilizer mixers, the optimal value " $m$ " corresponds to values of 4.0-5.0 [7].

The above mentioned hydraulic parameters of the mixer are directly related to its geometric parameters. The main defining condition for the mixer operation is the value of the created reduced header pressure $\mathrm{H}_{\mathrm{g} . p r}$, the maximum value of which for jet mixers was determined earlier and corresponds to the value of $20-25 \mathrm{~m}$. (This header pressure is created by the device of this type and was previously determined with all optimal geometric and hydraulic parameters). 
When calculating the mixer header pressure, it is necessary to keep in mind the fact that the header pressure of the mixer shall exceed the header pressure in the network by at least 3-5 m. Before the theoretical calculation of the required header pressure in the mixer, it is necessary to calculate the required header pressure of the network $H_{g . p r}$, determined by the dependence

$$
H_{2 . n p}=H_{2}+\sum h_{w c}
$$

where $H_{g}$ is the geometric height of the mixture supply by the mixer;

$$
\sum h_{w c} \text {-pressure losses in the system. }
$$

It is required to have in mind that the value of $H_{g . p r}$ depends on the technology used for irrigation.

The above-described dependence is suitable for use in case of using drip irrigation under the condition that the value of the header pressure loss $\Sigma h_{\mathrm{w}}$, the required pressure for the operation of drip lines is $\approx 20-25 \mathrm{~m}$.

When using other types of irrigation equipment, it is necessary to increase the design header pressure by the amount of free header pressure, providing for the operation of the machine used. For all hydraulic parameters of the mixer, an important role is played by the working flow rate in the nozzle $V_{0}$, which depends on the pressure of the working water flow generated by different types of sources (centrifugal nozzles). The header pressure flow rate in the nozzle is determined by the known dependence [8]

$$
V_{0}=\varphi \sqrt{2 g H_{H . n p}}
$$

where $\varphi$ is the rate coefficient (taken in this case 0.8 );

$H_{h . p r}$ is given header pressure of a centrifugal pump (working header pressure).

$\frac{V_{0}^{2}}{2 g}$

The $V_{0}$ value plays the main role in the creation of kinetic energy in the nozzle $\overline{2 g}$.

When the rate $V_{0}$ is increased to the maximum, the kinetic energy also increases to the

maximum (limited by cavitation phenomena in the nozzle) and the total energy $\frac{V_{0}^{2}}{2 g}+\frac{p_{0}}{g \rho}$, having passed the zero threshold, the cross section 0-0 (Figure 7) becomes negative, which contributes to the creation of vacuum either in the nozzle or in the mixing chamber. 


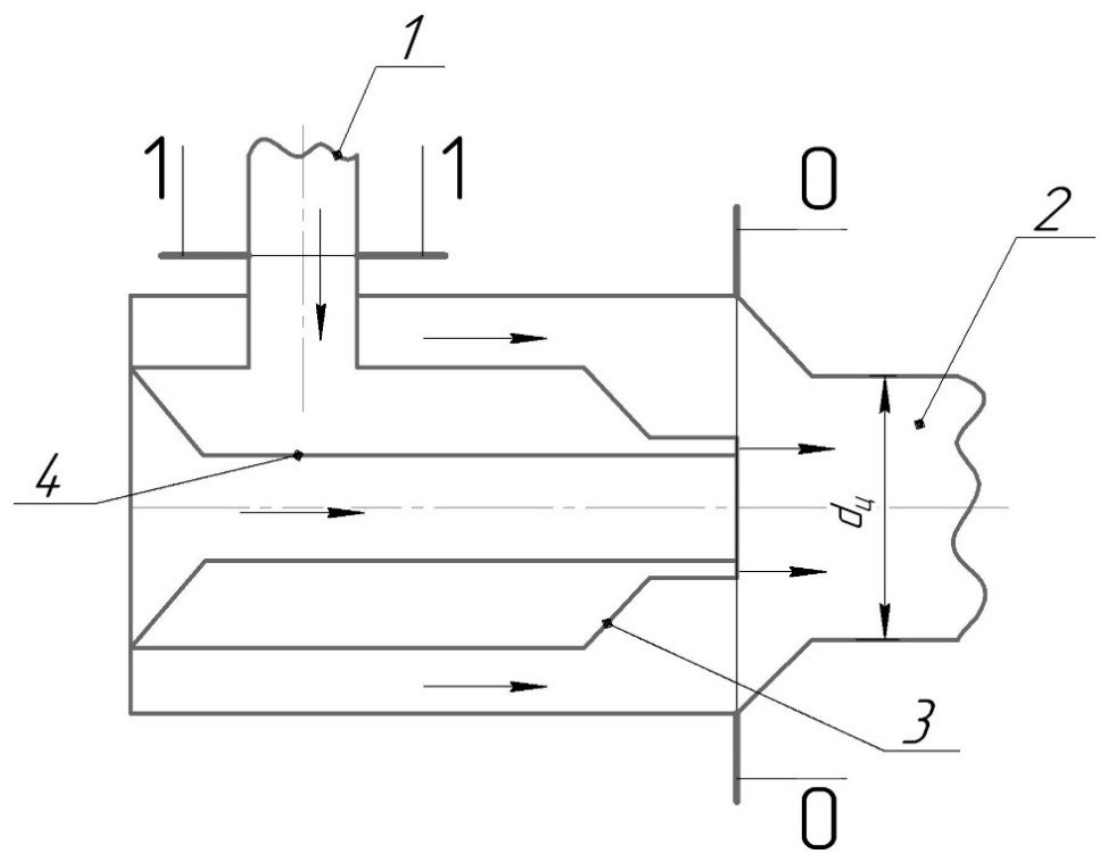

Fig. 7. Scheme of the working flow in the annular mixer.

Were: 1 - working water supply pipeline; 2 - mixing chamber; 3 - outer adapter; 4 - inner adapter.

The total energy in the cross-section $0-0$ is determined by drawing up the D. Bernoulli equation for the cross-sections " $1-1$ " and " $0-0$ ". For cross-section $1-1$, the total energy is calculated according to the dependence $\frac{p_{1}}{g \rho_{0}}+\frac{V_{1}^{2}}{2 g}$. . cause kinetic energy is $\frac{V_{1}^{2}}{2 g}$ $g$. As a rule, this energy is positive, bepositive and insignificant $(0.2-0.3 \mathrm{~m})$ due to low speed $(2-2.5$

$$
\underline{p_{1}}
$$

$\mathrm{m} / \mathrm{s})$ and high pressure $g \rho_{0} \quad(30-40-50 \mathrm{~m})$, depending on the parameters of the centrifugal pump. When the jet enters the nozzle, the rate is low $V$ and turns into the higher rate $V_{0}$ (up to $30-40 \mathrm{~m} / \mathrm{s}$ ). In this case, the kinetic energy increases to values of $45.8-82 \mathrm{~m}$ and changes in the "0-0", $\frac{p_{0}}{g \rho_{0}}+\frac{V_{0}^{2}}{2 g}$ cross-section: the value will $\frac{p_{0}}{g \rho_{0}}$ sharply fall, and the value $\frac{V_{0}^{2}}{2 g}$

sharply increases, but its total value will remain the same, equal $\frac{g \rho_{0}}{2 g}$, minus the header pressure losses in the portion from the cross-section 1-1 to cross-section $0-0$, determined by the dependence $\varsigma_{0} \frac{V_{0}^{2}}{2 g}$, where $\varsigma_{0}$ is the coefficient of hydraulic resistance 
of the nozzle (as a rule, this value for nozzles of this kind is equal to $0.06-0.10$ [9]). When considering these cross-sections according to the D. Bernoulli equation

$$
\frac{p_{1}}{g \rho_{0}}+\frac{V_{1}^{2}}{2 g}=\frac{p_{0}}{g \rho_{0}}+\frac{V_{0}^{2}}{2 g}+\varsigma_{0} \frac{V_{0}^{2}}{2 g}
$$

the potential energy in the nozzle is determined $\frac{p_{0}}{g \rho_{0}}$

$$
\frac{p_{0}}{g \rho_{0}}=\frac{p_{1}}{g \rho_{0}}+\frac{V_{1}^{2}}{2 g}-\frac{V_{0}^{2}}{2 g}-\varsigma_{0} \frac{V_{0}^{2}}{2 g}
$$

And as a result of the increased value $\frac{V_{0}^{2}}{2 g}$, the potential energy $\frac{p_{0}}{g \rho_{0}}$ will obviously become negative, the maximum value of which reaches $9.5 \mathrm{~m}$.

The value of such a vacuum is considered deep, which differs from the value of the vacuum in the suction pipelines of centrifugal pumps by almost 2-2.5 times. This calculation proves the superiority of the jet mixer suction capacity in comparison with the suction capacity of all types of pumping equipment.

When the flow enters the mixing chamber, the rate and kinetic energy decrease due to a change in the header pressure rate in the mixed flow. But it is required to have in mind that complete mixing of the working and suction flows occurs not at the beginning, but at the end of the mixing chamber. In jet mixers with a central supply, the velocity curve is elongated (Figure 8) and for complete mixing of flows, in such mixers the length of the mixing chamber, in contrast to the length of the mixing chamber of annular mixers (1.5-2 chamber diameters), is assumed to be 6-7 diameters, which when increased velocities play a significant role in both the total header pressure loss and the efficiency of the mixer.

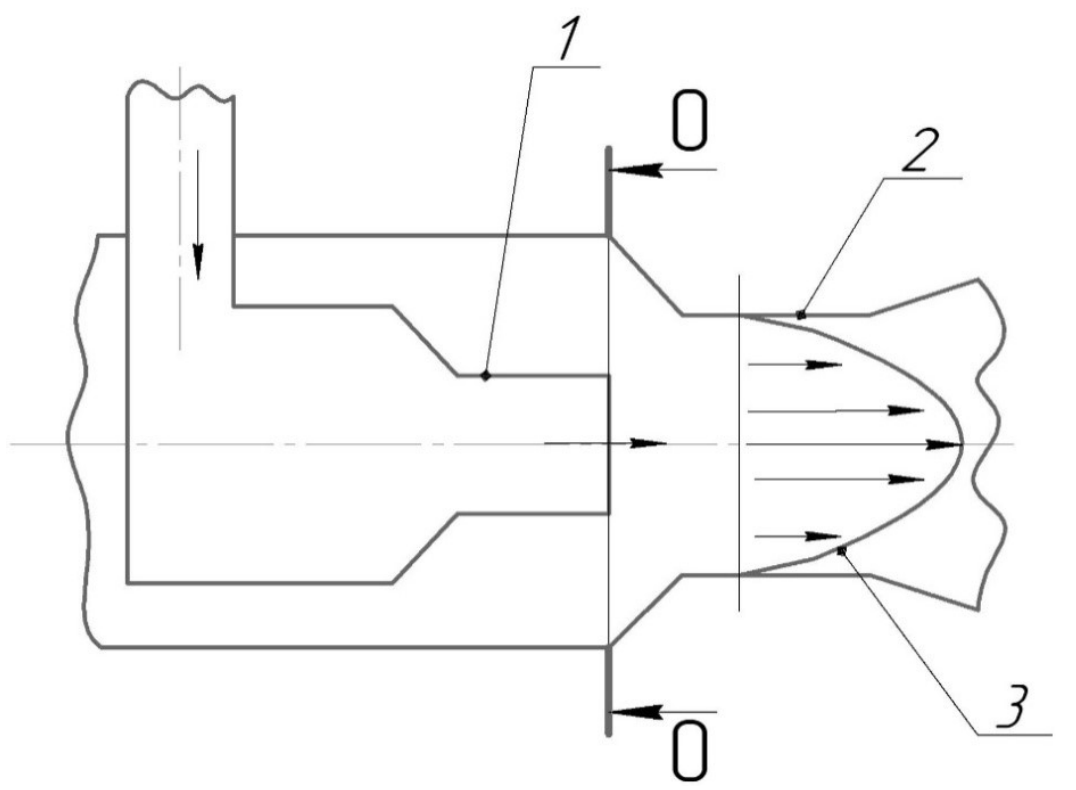


Fig. 8. Velocity curve in the mixing chamber of a mixer with a central inlet.

Were: 1 - nozzle; 2 - mixing chamber; 3 - velocity curve in the mixing chamber.

The mixing chamber in the existing designs of mixers begins at the nozzle end, in the cross-section $0-0$, in which two flows are actually transported: calculated and sucked in, with different values of kinetic and potential energy. The kinetic energy of the working flow, as mentioned above, has a value that exceeds the kinetic energy of the sucked flow by many times due to the high difference in velocities and to determine the boundary of the transition of high vacuum to medium (vacuum in a mixed flow) in the mixing chamber, it is necessary to carry out special investigations, not carried out so far. According to the literature data, experiments $[10,11]$ show that the kinetic energy from the zero cross-section to the average length of the mixing chamber remains high, and its value differs from the energy in the $0-0$ cross-section by $1-1.5 \%$, which indicates a high suction capacity mixer and high vacuum in the suction lines. The rate $V_{m . c}$ and the rate header pressure in the mixing chamber $\frac{V_{K . c}^{2}}{2 g}$ the two flows $\alpha_{0}$. At a low value of $\alpha_{0}(0.1,0.2)$, there is practically no suction flow, and the velocity in the mixer is the ratio of the working flow rate to the cross-sectional area of the mixing chamber

$$
V_{c}=\frac{Q_{0}}{\omega_{c}}
$$

With an increase in the coefficient $\alpha_{0}$ to the value of $1.0 ; 2.0 ; 3.0 ; 4.0$, etc. the sucked flow rate increases and the velocity in the mixing chamber is determined not by the ratio of the working flow rates $Q_{0}$, but by the ratio of the total flow rate $Q_{1}+Q_{0}$ to the area $\omega_{s}$.

$$
V_{c}=\frac{Q_{1}+Q_{0}}{\omega_{c}}
$$

Accordingly, with an increase in the flow rate in the mixing chamber, the amount of kinetic energy will also change $\frac{V_{c}^{2}}{2 g}$. At some values, $\alpha_{0}$ can become equal to the value of the kinetic energy in the nozzle $\frac{V_{0}^{2}}{2 g}$, in this case the values of the potential energy and vacuum in the mixing chamber $\frac{p_{c}}{g \rho_{0}}$ can become identical with the value of the potential energy in the nozzle $\frac{p_{0}}{g \rho_{0}}$.

When calculating the vacuum values in the suction pipelines, it is required to have in mind that if it is necessary to have a high suction header pressure in the mixer (suction vacuum), the mixer header pressure $H_{g . p r}$ should be calculated in such a way that the value of $\alpha_{0}$ does not decrease below 1.0. 
On the basis of the above mentioned recommendations, the possibility of determining the basic geometric and hydraulic parameters of a four-component jet mixer of fertilizers and water and supplying the mixture to irrigated areas during fertilizing irrigation has been developed, which will allow supplying all types of fertilizers and changing the mixture concentration in the required proportions.

\section{Conclusions}

The developed calculation bases are recommended for the theoretical substantiation of the geometric and hydraulic parameters of a four-component jet mixer of fertilizers and water during fertilizing irrigation. Recommendations for calculation are given for relative dimensions in case it is necessary to scale mixers for different options of their use. As the main parameter, the geometric characteristic of the mixer " $\mathrm{m}$ " shall be taken, in the range of 2.5 $\div 8.0$ corresponding to the maximum efficiency of the mixer up to $40 \%$.

\section{Reference}

1. Pat. No. 193355. Russian Federation, MPK A01 C23/04, Four-component jet pump mixer / V.A. Rudakov, K.A. Degtiareva, Yu.S. Urzhumova, S.A. Tarasyants; applicant and patentee State Federal-Funded Educational Institution of Higher Professional Training "Don State Agrarian University" - No. 2019111839; declared 18/04/2019; published on 25/10/2019, Bull. No. 12

2. V.E. Fridman, Hydroelevators, 142 (1990)

3. Kh.Sh. Mustafin, Scientific and technical communication, 10, 28 (1963)

4. Kh.Sh. Mustafin, Calculation of the ejector on water and slurry: a collection of works, 124 (1968)

5. Yu.L. Kirillovsky, Energy balance and calculation of water-jet devices, 23 (1957)

6. A.D. Altshul, Hydraulic resistance, 2, 224 (1982)

7. S.P. Ogorodnikov, Suction dredger injection, 48 (1962)

8. P.G. Kiselev and others, Guidance of hydraulic calculations, 352 (1972)

9. N.A. Rzhanitsyn, Water jet pumps, (1988)

10. N.A. Rzhanitsyn, Water jet pumps, 176 (1980)

11. S.A. Tarasyants, Environmentally sound technology and technical means for the preparation of livestock sewage for irrigation of forage crops, 44 (1995) 